\title{
ON SECONDARY VORTICITY IN INTERNAL WAVES*
}

\author{
BY \\ B. D. DORE AND M. A. AL-ZANAIDI $†$ \\ University of Reading, England
}

\begin{abstract}
Summary. The generation of secondary vorticity in maintained and temporallydecaying wave motions is investigated for internal waves in fluid of great depth. In the case of two semi-infinite, homogeneous fluids of different density, the interfacial boundary layers generate a second-order, mean vorticity which diffuses inwards into the interior of both fluids, and the net vorticity produced is zero. For a continuously-stratified fluid, the free surface layer plays an indirect role and secondary vorticity, initially generated only within stratified regions by the action of a Reynolds stress, diffuses, in general, over the whole fluid, and no steady-state vorticity field is established. In finite depths, a steady state ultimately exists for maintained waves, and the mass transport velocity field is investigated.
\end{abstract}

1. Introduction. It is well known in the small-amplitude theory of gravity waves, propagating on the surface of homogeneous fluid of great depth, that the first-order, oscillatory motion is irrotational except within a thin boundary layer adjacent to the free surface. The thickness of this layer is $O\left((\nu / \sigma)^{1 / 2}\right)$, where $\nu$ denotes the kinematic viscosity and $2 \pi / \sigma$ represents the wave period. But, in considering two-dimensional motion to second order in terms of the maximum wave slope, Longuet-Higgins [1] showed that secondary vorticity, generated within the free-surface boundary-layer, diffuses deeper and deeper into the interior of the fluid. At any fixed level in the fluid, the value of this vorticity ultimately becomes equal to the constant value at the edge of the free-surface boundary layer. Thus, secondary vorticity occurring in the interior of the fluid is generated by means of diffusive processes acting whilst the upper boundary - the edge of the surface layerhas this constant value. It is implicit in Longuet-Higgins' work that no second boundary layer, adjacent to the oscillatory, free-surface layer, exists. At sufficiently large times after initiation of the wave motion, and if such a second layer eventually exists, a further restriction may be required on the allowable wave amplitudes of the theory. Using a momentum method for short waves decaying with time, Phillips [2, pp. 38-40] gave a simple means of determining the value of the secondary vorticity at the edge of the surface layer, although this value had earlier been obtained in an elegant manner by LonguetHiggins [3]. Longuet-Higgins [4] discussed how the Lagrangian distribution of momentum of the decaying wave is transferred to the fluid in the form of a second-order shear current.

In the present work, the above results of Longuet-Higgins [1, 4] and Phillips [2] are

* Received January 17, 1977.

† Present address: Department of Applied Mathematics, University of Waterloo, Waterloo, Ontario, Canada N2L 3G1. 
extended to the case of density-stratified fluids of infinite depth. Thus, in Sec. 3, we consider a progressive internal wave propagating along the interface between two semiinfinite, homogeneous, immiscible fluids. In particular, it is shown how the momentum of a decaying wave is transferred to the fluids as a secondary current, and how secondary vorticity, generated within oscillatory, interfacial boundary layers of a wave which is maintained both spatially and temporally, penetrates the two fluids more and more through the action of viscous diffusion. These results are an extension of the work of Dore [5] for secondary, mean motions due to progressive waves in a two-layer system of finite depth.

Consideration is given in Sec. 4 to the creation of secondary vorticity induced by internal waves in continuously-stratified fluid of great depth. The Boussinesq approximation is made, in which buoyancy effects of density stratification are retained and inertial effects are neglected; also, the vertical motion of the free surface is assumed to be negligible, as discussed by Phillips [2, pp. 164-165]. The primary, oscillatory motion is itself rotational wherever stratification occurs, whilst the secondary vorticity possesses a fluctuating contribution, akin to a second harmonic, as well as a term which ultimately tends to a constant value at any fixed level. In the case of maintained waves, the value of the secondary vorticity at the edge of the oscillatory, free-surface boundary layer is zero. However, it transpires that, wherever the interior stratification is non-vanishing, there exists a Reynolds stress (of relevant order of magnitude) which acts to generate secondary vorticity within the entire fluid. The theory is applied to a simplified model of both a shallow and a deep oceanic thermocline, and graphs are presented to illustrate the results in the two cases.

Lastly, in Sec. 5, the mass transport velocity field is calculated for small-amplitude waves in the case of continuously-stratified fluid of finite depth, for which a steady-state vorticity field ultimately exists.

2. Formulation and existing results. We consider two-dimensional wave motion in incompressible, viscous fluids of infinite depths, and refer the equations to Cartesian coordinates $\left(x^{\prime}, y^{\prime}, z^{\prime}\right)$ for which the $z^{\prime}$-axis is directed vertically upwards. For continuously-stratified fluid, the origin lies in the mean free-surface level. When internal waves propagate along the interface separating homogeneous, immiscible fluids of great depths, the origin is taken in the mean interfacial level. Let $k$ denote wave number, $\sigma$ wave frequency, $\mathbf{q}^{\prime}$ and $\mathbf{r}^{\prime}$ the velocity and position vectors, $t^{\prime}$ the time, $\nu$ a kinematic viscosity, and $g$ the acceleration due to gravity. We introduce the non-dimensional variables

$$
\hat{\mathbf{r}}=k \mathbf{r}^{\prime}, \quad \hat{t}=\sigma t^{\prime}, \quad \hat{\mathbf{q}}=(k / \sigma) \mathbf{q}^{\prime}, \quad \epsilon=\left(\nu k^{2} / \sigma\right)^{1 / 2},
$$

and, in what follows, we shall mostly omit the symbol ( $\left.{ }^{\wedge}\right)$.

The layered system has densities $\rho^{(2)}$ (upper), $\rho^{(1)}$ (lower) and is stably-stratified $\left(\rho^{(1)}>\right.$ $\left.\rho^{(2)}\right)$. The continuously-stratified fluid has a statically-stable density distribution $\rho_{0}(z)$, and its motion is assumed to satisfy the conditions of the Boussinesq approximation. We write $\mathbf{q}=(u, 0, w)$ and define a stream function $\psi$ such that $u=\partial \psi / \partial z, w=-\partial \psi / \partial x$. In theories of waves of small amplitude, the velocity vector is expanded as

$$
\mathbf{q}=\alpha \mathbf{q}_{1}+\alpha^{2} \mathbf{q}_{2}+\cdots,
$$

in which $\alpha$ is an ordering parameter, and $\mathbf{q}_{1}$, etc., satisfy the linearized, etc., dynamical equations and boundary conditions. For a single progressive wave, we write

$$
\left(\psi_{1}, u_{1}, w_{1}\right)=(\Psi(z), U(z), W(z)) \exp (i(k x-\sigma t)),
$$


where only the real part of complex quantities associated with physical variables is to be understood.

When $\epsilon \ll 1$, it is well known that boundary layers of thickness $O(\epsilon)$ occur adjacent to the free surface or interface, and this inequality is assumed in the following work.

Let us first consider generation of secondary vorticity, associated with $\mathrm{q}_{2}$, at a clean free surface bounding homogeneous fluid of great depth. Before a progressive wave is set up, the vorticity $\omega=$ curl $\mathbf{q}$ is everywhere zero. Since vorticity cannot be generated within the fluid, the motion beyond the boundary layer (just after the waves are started) is irrotational and given by inviscid theory. An oscillatory, free-surface boundary layer is quickly established, within a few cycles of the initiation of the motion. Periodic vorticity $O(\alpha)$ exists within this layer, but decays to zero at the outer edge of the layer. Phillips [2] considers a progressive wave which decays with time. Since the Eulerian, mean wave momentum $M_{e}=E$ (= non-dimensional, mean energy density per unit surface area), dissipation of wave energy (mostly occurring in the interior) corresponds to a decrease in $M_{e}$. But, in an Eulerian description, the mean wave momentum is contained in the region above the horizontal level through the wave troughs. Decrease of $M_{e}$ must therefore be associated with a mean stress $S$ across horizontal planes just below this level, with $S=M_{e}$. $=E^{\circ}$. But the second-order Reynolds stress $-\overline{u_{1} w_{1}}$, where the bar denotes an average over a wave period, vanishes outside the oscillatory, free-surface layer. Hence, a mean, second-order viscous stress

$$
S=-\alpha^{2} \epsilon^{2} \partial \bar{u}_{2} / \partial z
$$

must be established to balance the loss of mean wave momentum. Then

$$
\bar{\omega}_{2}=\partial \bar{u}_{2} / \partial z=-S / \alpha^{2} \epsilon^{2}=-E^{\cdot} / \alpha^{2} \epsilon^{2} \quad \text { on } z=0 .
$$

But $E=\frac{1}{2} \alpha^{2} \exp \left(-4 \epsilon^{2} t\right)$ (Phillips [2], pp. 27, 37), so that $E^{\cdot} / E=-4 \epsilon^{2}$ and

$$
\bar{\omega}_{2}=-S / \alpha^{2} \epsilon^{2}=2 \exp \left(-4 \epsilon^{2} t\right) \quad \text { on } z=0 .
$$

This agrees with the original calculation of Longuet-Higgins [3, 1], and shows that mean vorticity $O\left(\alpha^{2}\right)$, generated within the surface layer, exists at the outer edge of this layer.

In connection with the previous paragraph, Longuet-Higgins [1] obtained the distribution of vorticity (corresponding to equation (2.5)) in the interior of the fluid. He assumed that the progressive wave decays with distance $x$, although it might, alternatively, be maintained spatially and temporally by suitable wind forces. The distribution of vorticity outside the surface layer is obtained by solving an initial-value problem for the diffusion equation. It is found that the secondary vorticity is given by $2\left[1-\operatorname{erf}\left(z / 2 \epsilon t^{1 / 2}\right)\right]$, and so, for a fixed value of $z$, tends to the value 2 as $t \rightarrow \infty$. Thus, mean, second-order vorticity diffuses deeper and deeper into the fluid, and no steady-state vorticity field $\bar{\omega}_{2}$ is established throughout the fluid.

Decay of a progressive wave. Longuet-Higgins [4] also discussed the case of slow, temporal decay of progressive surface waves in deep water, and sought the redistribution of the "initial" Langrangian distribution of wave momentum

$$
\alpha^{2} U_{s}=d M_{e} / d z_{0}=\alpha^{2} \exp \left(2 z_{0}\right),
$$

where $\alpha^{2} U_{s}$ is the horizontal component of the Stokes drift velocity

$$
\alpha^{2} \mathbf{Q}_{s}=\alpha^{2}\left(\overline{\int^{t} \mathrm{q}_{1} d t^{\prime} \cdot \nabla}\right) \mathrm{q}_{1} .
$$


Due to the viscous stress $S$ of Eq. (2.4), termed a "virtual tangential stress" by LonguetHiggins [1, 4], the decaying waves transfer all their momentum towards the boundary layer at the free surface. For the total momentum, $\tilde{M}$, transferred to the fluid by the stress $S$ during wave decay, is given by

$$
\tilde{M}=-\int_{0}^{\infty} S d t=\int_{0}^{\infty} 2 \alpha^{2} \epsilon^{2} \exp \left(-4 \epsilon^{2} t\right) d t=\frac{1}{2} \alpha^{2}=\left.M_{e}\right|_{t=0},
$$

so that all the momentum is transferred from the wave to the "mean flow" by the stress $S$. Corresponding to the "initial" distribution $\alpha^{2} U_{s}$, Longuet-Higgins solves an initial-value problem for the diffusion equation to find the subsequent distribution $\alpha^{2}\left(U_{s} \exp \left(-4 \epsilon^{2} t\right)+\right.$ $\left.u_{m}\right)$, where

$$
\alpha^{2} u_{m}=-\int_{0}^{t} f\left(t-t_{1}\right) S\left(t_{1}\right) d t_{1}
$$

and $f(t)=\exp \left(-z^{2} / 4 \epsilon^{2} t\right) /\left(\pi \epsilon^{2} t\right)^{1 / 2}$. Thus, for $t=O\left(\epsilon^{-2}\right)$, the decay time-scale of the wave, $u_{m}$ extends over those depths $O(1)$ affected by the original surface wave. For larger values of $t$, the depth affected increases as $\left(\epsilon^{2} t\right)^{1 / 2}$. Thus, in summary, we have the following features. (i) Initially, the momentum is entirely that due to the wave, (ii) for "moderate" values of $t$, less than $O\left(\epsilon^{-2}\right)$, momentum is being forced upwards during the decay time, (iii) for larger values of $t$, momentum diffuses downwards due to the action of viscosity.

The above results of Longuet-Higgins [1,4] and Phillips [2] will be extended in Sec. 3 to the case of an internal progressive wave on the interface between two homogeneous fluids of infinite depths, and, in Sec. 4, to the case of internal waves in a continuously-stratified fluid.

3. Progressive interfacial waves. We consider a progressive internal wave on the interface between two homogeneous fluids of great depths. The upper (lower) fluid has density $\rho^{(2)}\left(\rho^{(1)}>\rho^{(2)}\right)$ and kinematic viscosity $\nu^{(2)}\left(\nu^{(1)}\right)$. For each fluid, the vorticity equation has the form

$$
\partial \omega / \partial t+\partial(\omega, \psi) / \partial(x, z)=\epsilon^{2} \nabla^{2} \omega
$$

and we assume that $\epsilon^{(r)} \ll 1(r=1,2)$, so that there are boundary layers of thickness $O(\epsilon)$ adjacent to the oscillating interface.

(i) Generation of mean vorticity at the interface. Following Phillips' discussion as summarized in Sec. 2, we consider a progressive interfacial wave which decays with time. It is readily shown that $M_{e}=E$, and so energy dissipation (which now occurs predominantly in the interfacial boundary layers) corresponds to a reduction in $M_{e}$. In the Eulerian description, mean wave momentum is contained in the region between horizontal levels through interfacial crests and troughs. Any decrease in this momentum must be related to a net, mean horizontal force acting on the fluid between these levels. Thus, in dimensional terms,

$$
S^{(1)}+S^{(2)}=M_{e}^{\cdot}=E^{\cdot} / c,
$$

where $c=\sigma / k=$ wave speed, and $S^{(r)}$ is the component of this force acting in the positive $x$-direction. It is assumed that the interface is uncontaminated, and it can again be shown that the Reynolds stress $-\overline{u_{1} w_{1}}$ vanishes outside the interfacial boundary layers. Hence, there must be a change in the mean, second-order viscous stress across the whole interfacial boundary layer region,

$$
S=S^{(1)}+S^{(2)}=\alpha^{2}\left(-\mu^{(1)} \partial \bar{u}_{2}^{(1)} / \partial z+\mu^{(2)} \partial \bar{u}_{2}^{(2)} / \partial z\right),
$$


to balance the reduction in wave momentum. Then

$$
\mu^{(1)} \bar{\omega}_{2}^{(1)}-\mu^{(2)} \bar{\omega}_{2}^{(2)}=\left[\mu \bar{\omega}_{2}\right]=-E^{\cdot} / \alpha^{2} c \quad \text { on } z=0
$$

represents the change in $\mu \bar{\omega}_{2}$ across the whole interfacial boundary-layer region. But, if $a$ denotes the interfacial wave amplitude,

$$
\begin{gathered}
E=\frac{1}{2} a^{2} \sigma^{2} k^{-1}\left(\rho^{(1)}+\rho^{(2)}\right) \exp (-2 t / \tau), \\
\tau=\frac{\rho^{(1)}+\rho^{(2)}}{\left(2 \sigma k^{2}\right)^{1 / 2}} \frac{\left(\rho^{(1)} \mu^{(1)}\right)^{1 / 2}+\left(\rho^{(2)} \mu^{(2)}\right)^{1 / 2}}{\left(\rho^{(1)} \rho^{(2)} \mu^{(1)} \mu^{(2)}\right)^{1 / 2}},
\end{gathered}
$$

(see, for example, Dore [6]), so that

$$
\begin{gathered}
-E^{\cdot} / E=2 / \tau=O(\epsilon \sigma), \\
-S(t)=\alpha^{2}\left[\mu \bar{\omega}_{2}\right]=a^{2} \sigma \tau^{-1}\left(\rho^{(1)}+\rho^{(2)}\right) \exp (-2 t / \tau) \quad \text { on } z=0 .
\end{gathered}
$$

The quantity $S(t)$ may be termed "the net virtual tangential stress," by analogy with Longuet-Higgins [1, 4]. The result of Eq. (3.7) agrees with the direct mathematical calculation of Dore [5]. In non-dimensional terms, there must therefore be a change $O\left(\alpha^{2} \epsilon^{-1}\right)$ in the mean vorticity across the (oscillatory) interfacial boundary layers. This shows that such layers give rise to mean vorticity which is (formally) of greater intensity than that associated with boundary layers adjacent to clean free surfaces.

(ii) Decay of a progressive interfacial wave. An extension of the argument of LonguetHiggins [4] can be used to determine the momentum distribution in the interior of the two fluids, during the slow temporal decay of an internal wave propagating along the interface between two homogeneous layers of great depths. From a Lagrangian viewpoint, the initial distribution of wave momentum is

$$
\alpha^{2} \rho^{(r)} U_{s}^{(r)}=d M_{e}^{(r)} / d z_{0}=a^{2} \sigma k \rho^{(r)} \exp \left(2 j k z_{0}\right)\left(\begin{array}{lll}
j=1 & \text { if } & r=1 \\
j=-1 & \text { if } & r=2
\end{array}\right) .
$$

Due to the virtual stress of Eq. (3.7), the waves transfer all their momentum (during the decay time $O(\tau))$ towards the boundary layers at the interface. The total momentum is

$$
\tilde{M}=-\int_{0}^{\infty} S(t) d t=\frac{1}{2} a^{2} \sigma\left(\rho^{(1)}+\rho^{(2)}\right)=M_{e}^{(1)}+M_{e}^{(2)},
$$

so that all the wave momentum is transferred to the mean flow by the interfacial stress $S(t)$. To find the distribution of this mean flow in both fluids, we need to solve the following initial-value problem. In each fluid,

$$
\partial \omega_{2}^{(r)} / \partial t=\nu^{(r)} \partial^{2} \omega_{2}^{(r)} / \partial z^{2}
$$

since convection effects are zero, under the assumed wave conditions ${ }^{1}$. The boundary and initial conditions are as follows. The first-order motion is quickly established and, after a few oscillations, vorticity $O\left(\alpha^{2} \epsilon^{-1} \sigma\right)$ is set up in the oscillatory boundary layers, but has not penetrated the interior. Thus,

$$
\omega_{2}^{(r)}=0 \quad \text { for } t \leq 0, z \neq 0 .
$$

Far from the interface, we have

$$
\omega_{2}^{(r)} \rightarrow 0 \quad \text { as } z \rightarrow-j \infty, t \geq 0 .
$$

\footnotetext{
${ }^{1}$ It is assumed, as in the work of Longuet-Higgins [1], that no second boundary layer (adjacent to the freesurface oscillatory layer) exists. If, ultimately, there is such a layer, the solution determined on the basis of Eq. (3.9) breaks down at very large times after initiation of the wave motion.
} 
The interfacial conditions are as follows. Firstly, the vorticity satisfies

$$
\alpha^{2}\left(\mu^{(1)} \omega_{2}^{(1)}-\mu^{(2)} \omega_{2}^{(2)}\right)=a^{2} \sigma \tau^{-1}\left(\rho^{(1)}+\rho^{(2)}\right) \exp (-2 t / \tau) \quad \text { for } t \geq 0, z=0 .
$$

This may be deduced at any instant $t \geq 0$ by using a momentum argument similar to that of (i) above. Secondly, according to Dore [5], the change in the mean tangential velocity across the whole interfacial boundary-layer region, when in a steady state, is $O\left(\alpha^{2} c\right)$. This can be expected to hold for any $t \geq 0$, for otherwise the value of $\omega_{2}$ in the boundary layers would be very much greater than that suggested by Eq. (3.12). Hence, we take

$$
\begin{gathered}
u_{m}^{(1)}=u_{m}^{(2)} \quad \text { for } t \geq 0, z=0, \\
u_{m}^{(1)}=\int_{-\infty}^{z} \omega_{2}^{(1)} d z, \quad u_{m}^{(2)}=\int_{\infty}^{z} \omega_{2}^{(2)} d z .
\end{gathered}
$$

By Eqs. (3.9), (3.14), $u_{m}$ satisfies

$$
\partial u_{m} / \partial t=\nu \partial^{2} u_{m} / \partial z^{2}
$$

in each fluid. We now obtain the solution for $u_{m}$ by applying the Laplace transformation

$$
L\left\{u_{m}\right\}=\int_{0}^{\infty} \exp (-p t) u_{m}(z, t) d t,
$$

and find that

$$
\alpha^{2} L\left\{u_{m}\right\}=\frac{-L\{S\} \exp \left(j\left(p / \nu^{(r)}\right)^{1 / 2} z\right)}{p^{1 / 2}\left(\left(\rho^{(1)} \mu^{(1)}\right)^{1 / 2}+\left(\rho^{(2)} \mu^{(2)}\right)^{1 / 2}\right)} .
$$

Using the convolution theorem, we thus obtain

$$
\alpha^{2} u_{m}^{(r)}=-\int_{0}^{t} \frac{f^{(r)}\left(t-t_{1}\right) S\left(t_{1}\right)}{\left(\rho^{(1)} \mu^{(1)}\right)^{1 / 2}+\left(\rho^{(2)} \mu^{(2)}\right)^{1 / 2}} d t,
$$

where $f^{(r)}(t)=\exp \left(-z^{2} / 4 \nu^{(r)} t\right) /(\pi t)^{1 / 2}$. At time $t=O(\tau)$, the depths of the regions affected are $O\left(\epsilon^{1 / 2} k^{-1}\right)$. For larger times, the depths affected increase as $O\left([\epsilon t / \tau]^{1 / 2} k^{-1}\right)$. Thus, the momentum $\rho^{(r)} u_{m}^{(r)}$ per unit depth moves towards the interface during the decay time $\tau$, and is then diffused (by viscosity) to the interior of the upper and lower fluids. This momentum is, as shown above, equivalent to the original momentum of the wave. To the present order of working, the total amount of vorticity diffused downwards into the lower layer is, at any instant, equal and opposite to the total amount of vorticity diffused upwards into the upper layer. Thus, the total amount of vorticity in the whole system is zero.

(iii) Vorticity distribution for maintained waves. This may be obtained immediately from Eq. (3.16), by first omitting the exponential-decay term in Eq. (3.12). Then

$$
\alpha^{2} \omega_{2}^{(r)}=\frac{a^{2} \sigma j\left(\rho^{(1)}+\rho^{(2)}\right)}{\tau\left(\nu^{(r)}\right)^{1 / 2}\left(\left(\rho^{(1)} \mu^{(1)}\right)^{1 / 2}+\left(\rho^{(2)} \mu^{(2)}\right)^{1 / 2}\right)}\left[1-\operatorname{erf}\left(-j z / 2\left(\nu^{(r)} t\right)^{1 / 2}\right)\right],
$$

and

$$
\left.\sigma^{-1} \omega_{2}^{(r)}\right|_{2=0}=\frac{j}{\epsilon^{(r)}} \frac{\left(2 \rho^{(1)} \rho^{(2)} \mu^{(1)} \mu^{(2)}\right)^{1 / 2}}{\left(\left(\rho^{(1)} \mu^{(1)}\right)^{1 / 2}+\left(\rho^{(2)} \mu^{(2)}\right)^{1 / 2}\right)^{2}} .
$$

Thus, just as $\left[\mu \omega_{2}\right]$ is time-independent, so also are the individual values of the vorticity at the edges of the interfacial boundary layers. Moreover, for any fixed value of $z$, the 
vorticity $\omega^{(r)}(z, t) \rightarrow \omega^{(r)}(0, t)$ as $t \rightarrow \infty$. Therefore, as time increases, a mean, second-order vorticity field diffuses deeper and deeper into each fluid, and so no steady state of $\omega_{2}$ is established throughout the entire fluid system.

4. Continuously-stratified fluid. Next, we consider progressive internal waves in fluid of infinite depth which, in equilibrium, has the statically-stable and continuous density field $\rho_{d}(z)$. The non-dimensional form of the vorticity equation is

$$
\frac{\partial \omega}{\partial t}+\frac{\partial(\omega, \psi)}{\partial(x, z)}=\epsilon^{2} \nabla^{2} \omega+G \frac{\partial \hat{\rho}}{\partial x},
$$

where $G=g k / \sigma^{2}, \rho(\mathrm{r}, t)=\rho_{0}(0) \hat{\rho}$ and the Boussinesq approximation has been made. The incompressibility condition is

$$
\partial \rho / \partial t+\partial(\rho, \psi) / \partial(x, z)=0 .
$$

Before a progressive internal wave is set up, the vorticity is everywhere zero; but, immediately after the waves are started, the motion is rotational, wherever the buoyancy (BruntVäisälä) frequency $N(z)=\left[-g \rho_{0}^{\prime} / \rho_{0}(0)\right]^{1 / 2}$ is non-zero, and is given by inviscid theory. If $\epsilon$ $\ll<1$ and $N(z)$ is everywhere continuous, an oscillatory boundary layer of thickness $O(\epsilon)$ is quickly established at the free surface alone. Initially, and assuming, as Thorpe [7] did in a steady-state wave motion, that, outside this boundary layer, there is no mean, Eulerian current $\bar{u}_{2}$ due to the internal wave, it is readily shown that the net momentum $M_{e}=0$ (neglecting a very small free-surface effect). Moreover, it may also be readily shown that the mean, second-order equations within the surface layer may be integrated (to the present order of working) by assuming that the fluid there is locally homogeneous. Then Eq. (227) of Longuet-Higgins [3] yields

$$
\bar{\omega}_{2}=0
$$

at the edge of the oscillatory layer, since vertical motion at the free surface is assumed to be negligible. Thus, no secondary vorticity is generated at a clean free surface during such an internal wave motion. Nevertheless, it will be seen that Eq. (4.3) plays an important role in the determination of the secondary vorticity generated in the interior of the fluid.

(i) Generation of secondary vorticity for maintained waves. Assuming that the waves are maintained both spatially and temporally by the action of suitable forces (which, however, do not modify Eq. (4.3)), the oscillatory surface layer is established within a few cycles, and we have, approximately,

$$
\omega_{2}=F(z) \cos 2(k x-\sigma t+\chi) \quad \text { for } t=0, z \leq 0
$$

by our assumption about $\bar{u}_{2}$. Also, as in Sec. 3(ii), we have

$$
\omega_{2} \rightarrow 0 \quad \text { as } z \rightarrow-\infty, t \geq 0 \text {. }
$$

We are predominantly interested in the contribution to $\omega_{2}$ which, at any point $r$, tends to a limiting value as $t \rightarrow \infty$, and, hence, ultimately possesses a "mean" part. Therefore, we write

$$
\omega_{2}=\omega_{22}(x, z, t)+\omega_{m}(z, t)
$$

and confine our interest here to the creation of the secondary vorticity field $\omega_{m}$. By Eqs. 
(4.3)-(4.5), and by Appendix 1, $\omega_{m}$ is given by the solution of the initial-value problem

$$
\begin{aligned}
\partial \omega_{m} / \partial t-\epsilon^{2} \partial\left(\overline{\zeta_{1} \nabla^{2} \omega_{1}}\right) / \partial z=\epsilon^{2} \partial^{2} \omega_{m} / \partial z^{2}, \\
\omega_{m}=0 \quad \text { for } t \geq 0, z=0, \\
\omega_{m}=0 \quad \text { for } t \leq 0, z \leq 0, \\
\omega_{m} \rightarrow 0 \quad \text { as } z \rightarrow-\infty, t \geq 0,
\end{aligned}
$$

where $\zeta$ denotes the vertical displacement of a fluid element. It is seen in Appendix 1 that $\omega_{m}$ arises because of the action of a Reynolds stress $-\overline{u_{1} w_{1}}$, of order $\alpha^{2} \epsilon^{2}$, which (as in Secs. 2,3 ) vanishes wherever the stratification vanishes; in fact, the forcing term in the generalized diffusion equation (4.7) is

$$
-\epsilon^{2} \frac{\partial}{\partial z}\left(\overline{\zeta_{1} \nabla^{2} \omega_{1}}\right)=\frac{1}{2} \epsilon^{2} \frac{\partial}{\partial z}\left[\Psi\left(\frac{d^{2}}{d z^{2}}-1\right)\left(\frac{N^{2}}{\sigma^{2}} \Psi\right)\right]=\epsilon^{2} f(z),
$$

and so is known in terms of the linear inviscid quantity $\Psi(z)$. The solution of the initialvalue problem (4.7)-(4.10) is given by Carslaw and Jaeger [8, pp. 59, 79] as

$\omega_{m}=g(z)+\frac{1}{2 \epsilon(\pi t)^{1 / 2}} \int_{-\infty}^{0} g(Z)\left(\exp \left(-(z+Z)^{2} / 4 \epsilon^{2} t\right)-\exp \left(-(z-Z)^{2} / 4 \epsilon^{2} t\right)\right) d Z$,

where

$$
g(z)=-\frac{1}{2} \int_{z}^{0} \Psi\left(\frac{d^{2}}{d Z^{2}}-1\right)\left(\frac{N^{2}}{\sigma^{2}} \Psi\right) d Z=\frac{1}{2}\left[\left(\frac{N^{2}}{\sigma^{2}}\right)^{\prime} \Psi^{2}+\int_{z}^{0} \frac{N^{4}}{\sigma^{4}} \Psi^{2} d Z\right] .
$$

For fixed $t, \omega_{m} \rightarrow 0$ as $z \rightarrow-\infty$. But, for a fixed value of $z, \omega_{m} \rightarrow g(z)$ as $t \rightarrow \infty$ and therefore tends to a definite limiting value. For sufficiently large values of $|z|$,

$$
g \simeq \frac{1}{2} \int_{z}^{0} \frac{N^{4}}{\sigma^{4}} \Psi^{2} d Z
$$

which thus shows that time-independent vorticity, generated within stratified regions through the action of a Reynolds stress, diffuses deeper and deeper into the fluid and no steady state is established. The above theory holds for internal modes of any order.

An example. We consider the class of density distributions $\rho_{0}(z)$ corresponding to the thin-thermocline model described by Phillips [2, pp. 166-170]. The stratification is such that $N(z)=0$ except within a narrow region, within which $N$ has a single maximum. The model has possible validity in certain oceanic situations when the upper layer, above the permanent thermocline, has been vigorously mixed. Phillips used the assumed thinness of the thermocline, centered about the level $z=-d$, to discuss the character of the first internal mode. However, much of his approximate calculation is erroneous, and a valid approach, retaining a first correction for finite thickness $b$ of the thermocline, is given by Al-Zanaidi and Dore [9]. For present purposes, we write $N(z)=0$ where $0>z>-d_{+}$, $-d_{-}>z$ (so that $z=-d_{ \pm}$denote the top, bottom of the thermocline) and assume, at first, that $N(z)$ is everywhere continuous. We then find the expressions

$$
\begin{array}{ll}
g(z)=0 & \left(0>z>-d_{+}\right), \\
g(z) \simeq \frac{1}{2} \Psi_{d}^{2}\left[\left(\frac{N^{2}}{\sigma^{2}}\right)^{\prime}+\int_{z}^{-d_{+}} \frac{N^{4}}{\sigma^{4}} d Z\right] & \left(-d_{+}>z>-d_{-}\right), \\
g(z) \simeq \frac{1}{2} \Psi_{d}^{2} \int_{-d_{-}}^{-d_{+}} \frac{N^{4}}{\sigma^{4}} d Z & \left(-d_{-}>z>-\infty\right),
\end{array}
$$


where $\Psi_{d}$ is the (approximately) uniform value of $\Psi$ within the thermocline during the first internal mode. As shown in [9], this approximation requires that $b \ll 1$. For particular stratifications, equation (4.14) may be substituted into (4.12) to yield the secondary vorticity $\omega_{m}$. The simplest case, which we now consider, occurs when the thermocline is uniformly stratified $\left(N^{2}=N_{0}^{2}=\right.$ constant, there). Oscillatory boundary layers of thickness $O(\epsilon)$ are required adjacent to both the top and bottom of the thermocline, since the discontinuity in $N$ renders $\omega_{1}$ (but not $u_{1}$ ) discontinuous there, according to inviscid theory. These layers are very weak, and the vorticity within them is no larger than that within the interior of the thermocline. Thus, the nature of the layers is similar to that of a layer adjacent to a free surface not subject to any wind stress and not contaminated by a surface film. The top, bottom surfaces of the thermocline are regarded as "interfaces", at which the density $\rho_{0}$ is continuous but the density gradient $\rho_{0}^{\prime}$ is discontinuous. In the wave motion, the conditions of continuity of tangential stress and tangential velocity must be satisfied at the interfaces. The former condition is implicit in the following statement. Across each of the interior layers, there is no change in $\omega_{m}$ at any instant $t \geq 0$, so that

$$
\left[\omega_{m}\right]=0 \quad \text { for } t \geq 0, z=-d_{ \pm} .
$$

This may be immediately shown by use of the momentum argument of Secs. 2, 3(ii). The analogous condition on the horizontal velocity $u_{m}$ (corresponding to $\omega_{m}$ ) requires some considerable explanation. Briefly, because $N$ is discontinuous at the interfaces, the secondorder Stokes drift velocity $U_{s}$ of Eq. (2.7) is discontinuous, by an amount $\frac{1}{2}\left(N_{0}^{2} / \sigma^{2}\right) \Psi_{d}^{2}$, across the interior boundary layers ${ }^{2}$. By using curvilinear coordinates, it may be shown that $u_{m}$ is also discontinuous in the uniform wave motion-Al-Zanaidi [10]-by an amount which makes the mass transport velocity (see Sec. 5) continuous across the interior boundary layers. The extension to any $t \geq 0$ follows on the lines of the discussion on tangential velocity just above Eq. (3.13), so that we have

$$
\begin{array}{ll}
{\left[u_{m}\right]=\frac{1}{2}\left(N_{0}^{2} / \sigma^{2}\right) \Psi_{d}^{2}} & \text { for } t \geq 0, z=-d_{+}, \\
{\left[u_{m}\right]=-\frac{1}{2}\left(N_{0}^{2} / \sigma^{2}\right) \Psi_{d}^{2}} & \text { for } t \geq 0, z=-d_{-} .
\end{array}
$$

Consequently, for the present idealized density distribution, it is not possible to prescribe an initially-zero secondary current throughout the entire depth. Now, $u_{m}(z, t)$ satisfies the equation

$$
\partial u_{m} / \partial t-\epsilon^{2} \overline{\zeta_{1} \nabla^{2} \omega_{1}}=\epsilon^{2} \partial^{2} u_{m} / \partial z^{2}
$$

in each of the regions $0>z>-d_{+},-d_{+}>z>-d_{-},-d_{-}>z>-\infty$, together with the conditions (4.8), (4.9), (4.15), (4.16) and

$$
u_{m} \rightarrow 0 \quad \text { as } z \rightarrow-\infty, t \geq 0 .
$$

Due to the linearity of the problem for $u_{m}$, we may write

$$
u_{m}=u_{m 1}+u_{m 2} \text {, }
$$

where $u_{m 1}$ arises on account of the forcing function in Eq. (4.17), and satisfies homogeneous interfacial conditions, whilst $u_{m 2}$ satisfies the homogeneous diffusion equation and is

\footnotetext{
${ }^{2}$ If $a$ is the vertical displacement of the top of the thermocline during the first internal mode, the ordering parameter of Eq. (2.2) is taken to be $\alpha=a k$. Further, since $U_{s}$ arises from a Taylor series expansion about the equilibrium position of a fluid element, we require $a \ll b$ here.
} 
due to the non-homogeneous interfacial condition (4.16). Then, for $t \geq 0$,

$$
\begin{aligned}
u_{m 2} & =0 & & \left(0>z>-d_{+}\right), \\
& =\frac{1}{2}\left(N_{0}^{2} / \sigma^{2}\right) \Psi_{d}^{2} & & \left(-d_{+}>z>-d_{-}\right), \\
& =0 & & \left(-d_{-}>z>-\infty\right),
\end{aligned}
$$

and corresponds to a uniform flow in the thermocline in the direction of wave propagation. Conversely, $u_{m 1}$ is rotational and, it may be readily shown, corresponds to the solution (4.12) with

$$
\begin{array}{ll}
g(z)=0 & \left(0>z>-d_{+}\right), \\
g(z) \simeq-\frac{1}{2}\left(N_{0}^{4} / \sigma^{4}\right) \Psi_{d}^{2}\left(z+d_{+}\right) & \left(-d_{+}>z>-d_{-}\right), \\
g(z) \simeq \frac{1}{2}\left(N_{0}^{4} / \sigma^{4}\right) \Psi_{d}^{2} b & \left(-d_{-}>z>-\infty\right) .
\end{array}
$$

Thus, we find from Eq. (4.12) that

$$
\begin{aligned}
{\left[\omega_{m}-g(z)\right] /\left(\frac{1}{2} \frac{N_{0}^{2}}{\sigma^{2}} \Psi_{d}\right)^{2}=b\left[\operatorname{erf}\left(\frac{z+d_{-}}{2 \epsilon t^{1 / 2}}\right)+\operatorname{erf}\left(\frac{z-d_{-}}{2 \epsilon t^{1 / 2}}\right)\right] } \\
+\left(z-d_{+}\right)\left[\operatorname{erf}\left(\frac{z-d_{+}}{2 \epsilon t^{1 / 2}}\right)-\operatorname{erf}\left(\frac{z-d_{-}}{2 \epsilon t^{1 / 2}}\right)\right] \\
\quad-\left(z+d_{+}\right)\left[\operatorname{erf}\left(\frac{z+d_{+}}{2 \epsilon t^{1 / 2}}\right)-\operatorname{erf}\left(\frac{z+d_{-}}{2 \epsilon t^{1 / 2}}\right)\right] \\
-2 \epsilon(t / \pi)^{1 / 2}\left[\exp \left(-\left(z+d_{+}\right)^{2} / 4 \epsilon^{2} t\right)+\exp \left(-\left(z-d_{-}\right)^{2} / 4 \epsilon^{2} t\right)\right. \\
\left.-\exp \left(-\left(z-d_{+}\right)^{2} / 4 \epsilon^{2} t\right)-\exp \left(-\left(z+d_{-}\right)^{2} / 4 \epsilon^{2} t\right)\right] .
\end{aligned}
$$

This solution for the secondary vorticity field $\omega_{m}(z, t)$ associated with the lowest internal mode is illustrated for the case $d_{+}=4 b$ in Fig. 1, in which

$$
\Omega_{m}=\omega_{m} /\left(\frac{1}{2} \frac{N_{0}^{2}}{\sigma^{2}} \Psi_{d}\right)^{2} .
$$

It is clearly seen that such vorticity diffuses outwards from the thermocline and, as time increases, extends deeper and deeper into the lower homogeneous fluid. On the other hand, whilst the upper homogeneous fluid becomes rotational for finite times, it ultimately regains its irrotational character on account of the influence of the upper boundary condition (4.8). It is also noted that $\partial \omega_{m} / \partial z$ is discontinuous at the thermocline edges, and that $\omega_{m}$ is almost linear within the stratified region (except for very small values of the time).

The solution (4.20) is thought to be indicative of the corresponding solutions for $\omega_{m}$ when $N$ varies smoothly and is roughly constant over much of the thermocline. For such stratifications, the quantity $u_{m 2}$ is, of course, irrelevant.

If, for general density distributions, the internal wave motion is initially set up with $\omega_{m}$ $\neq 0$, the solution (4.12) represents the change in the corresponding vorticity field between $t$ $=0$ and $t=\infty$.

(ii) Generation of secondary vorticity in unbounded fluids. We also consider the particular case of a uniformly-stratified region separating two semi-infinite, homogeneous fluids. It is convenient now to take the origin of coordinates on a level midway between the top 


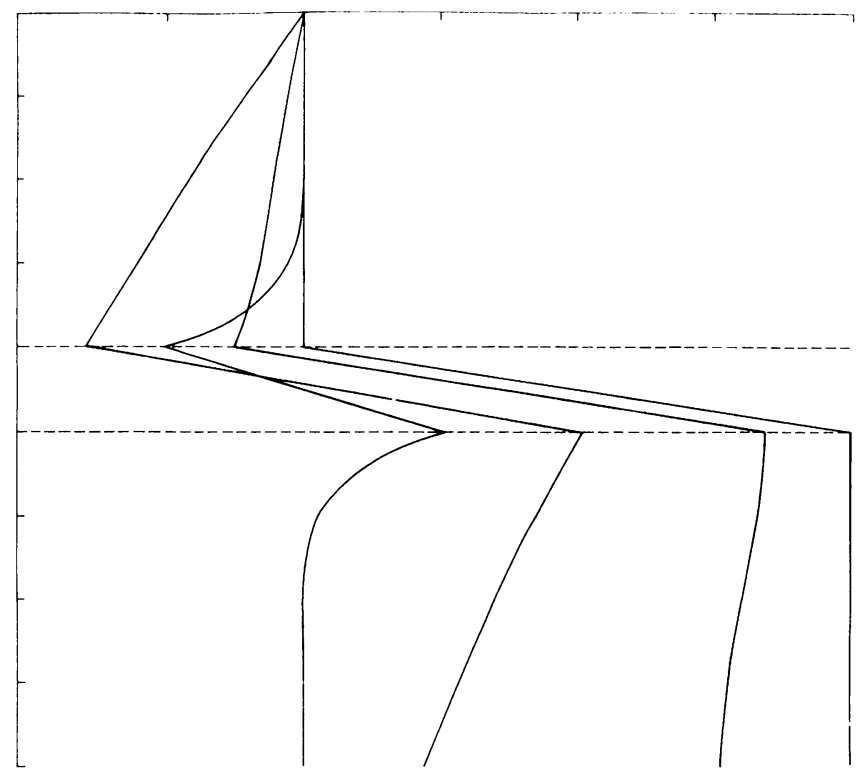

FIG. 1. Instantaneous distributions of secondary vorticity associated with the first internal mode on a thin, shallow thermocline occupying the region $-4 b>z>-5 b$.

and bottom of the thermocline. The boundary condition (4.8) must be replaced by the condition

$$
\omega_{m} \rightarrow 0 \quad \text { as } z \rightarrow \infty, t \geq 0 .
$$

Then the solution of the initial-value problem for the secondary vorticity $\omega_{m}$ associated with the first internal mode in a maintained wave motion is given by Carslaw and Jaeger $[8$, p. 53] as

$$
\omega_{m}=g(z)-\frac{1}{2 \epsilon(\pi t)^{1 / 2}} \int_{-\infty}^{\infty} g(Z) \exp \left(-(z-Z)^{2} / 4 \epsilon^{2} t\right) d Z
$$

where

$$
\begin{array}{rlrl}
g(z) & =-\frac{1}{2} \int_{z}^{\infty} \Psi\left(\frac{d^{2}}{d Z^{2}}-1\right)\left(\frac{N^{2}}{\sigma^{2}} \Psi\right) d Z, \\
& =0 & & \left(\infty>z>\frac{1}{2} b\right), \\
& \simeq-\frac{1}{2}\left(N_{0}^{4} / \sigma^{4}\right) \Psi_{d}^{2}\left(z-\frac{1}{2} b\right) & & \left(\frac{1}{2} b>z>-\frac{1}{2} b\right), \\
& \simeq \frac{1}{2}\left(N_{0}^{4} / \sigma^{4}\right) \Psi_{d}^{2} b & & \left(-\frac{1}{2} b>z>-\infty\right) .
\end{array}
$$

Then, from Eq. (4.22), we find that

$$
\begin{aligned}
& {\left[\omega_{m}-g(z)\right] /\left(\frac{1}{2} \frac{N_{0}^{2}}{\sigma^{2}} \Psi_{d}\right)^{2}=b\left[\operatorname{erf}\left(\frac{z+\frac{1}{2} b}{2 \epsilon t^{1 / 2}}\right)-1\right]} \\
& +\left(z-\frac{1}{2} b\right)\left[\operatorname{erf}\left(\frac{z+\frac{1}{2} b}{2 \epsilon t^{1 / 2}}\right)-\operatorname{erf}\left(\frac{z-\frac{1}{2} b}{2 \epsilon t^{1 / 2}}\right)\right] \\
& -2 \epsilon(t / \pi)^{1 / 2}\left[\exp \left(-\left(z-\frac{1}{2} b\right)^{2} / 4 \epsilon^{2} t\right)-\exp \left(-\left(z+\frac{1}{2} b\right)^{2} / 4 \epsilon^{2} t\right)\right],
\end{aligned}
$$


and that $\omega_{m}(z, t)$ is therefore an odd function of $z$. Consequently, as in Sec. 3(iii), the total amount of vorticity generated at any instant is zero. The solution for the secondary vorticity field in the lowest internal mode is shown in Fig. 2 for several values of the time. For small values of $t$, say $\epsilon t^{1 / 2} \leq \frac{1}{b}$, the solutions (4.20), (4.23) are practically identical. As $t$ increases, the vorticity extends more and more into each of the homogeneous fluids.

(iii) Decay of a progressive internal wave. When the Boussinesq approximation is applied and vertical motion of the free surface is neglected, the net, Eulerian, mean momentum of an internal wave is zero, $M_{e}=0$. Therefore, the question of transfer of net momentum, during temporal decay of the wave, does not arise. Nevertheless, it is still possible to derive an explicit formula for the generation of secondary vorticity. The decay time for an internal wave in infinite depth has been calculated by Dore [11] for general stratifications, and is such that $\tau=O\left(\epsilon^{-2}\right)$. By Appendix 2, the initial-value problem now becomes

$$
\begin{aligned}
\partial \omega_{m} / \partial t+\left[\epsilon^{2} f(z)+2 \tau^{-1}\left(\frac{N^{2}}{\sigma^{2}} \Psi^{2}\right)^{\prime}\right] \exp (-2 t / \tau)=\epsilon^{2} \partial^{2} \omega_{m} / \partial z^{2}, \\
\omega_{m}=0 \quad \text { for } t \geq 0, z=0, \\
\omega_{m}=0 \quad \text { for } t \leq 0, z \leq 0, \\
\omega_{m} \rightarrow 0 \quad \text { as } z \rightarrow-\infty, t \geq 0,
\end{aligned}
$$

where $f(z)$ is given by Eq. (4.11). The formal solution of this generalised diffusion equation may be obtained by use of Duhamel's superposition theorem, Carslaw and Jaeger [8, pp. $30-32]$, and is

$$
\begin{aligned}
\omega_{m}(z, t ; \tau) & =\frac{\partial}{\partial t} \int_{0}^{t} \Omega\left(z, t-t_{1}\right) \exp \left(-2 t_{1} / \tau\right) d t_{1} \\
& =\Omega(z, t)-2 \tau^{-1} \int_{0}^{t} \Omega\left(z, t_{1}\right) \exp \left(-2\left(t-t_{1}\right) / \tau\right) d t_{1},
\end{aligned}
$$

where $\Omega$ is the solution of a modified form of the above problem, in which the exponential factor in the forcing term of Eq. (4.24) is omitted. In particular, the specific problems considered for the uniformly-stratified, thin-thermocline model in (i), (ii), above, may be investigated as examples of decaying internal waves, and $\Omega$ has the same form as $\omega_{m}$ in Eqs. (4.20), (4.23). The integrals occurring in Eq. (4.25) can be evaluated analytically in terms of error functions of complex argument-see, for example, Abramowitz and Stegun [12, p. 304].

5. Mass transport velocity. When the stratified fluid has finite depth $h$, and the characteristic wave amplitudes are such that $\alpha \ll \epsilon$, a steady-state vorticity field is established at a time $O\left(h^{2} / \epsilon^{2}\right)$ after initiation of the wave motion. This is the time required for mean vorticity to diffuse over the whole fluid. It is of interest to calculate the mass transport, or Lagrangian mean, velocity $\alpha^{2} \mathbf{Q}_{l}$; that is, the velocity, averaged over a wave period, which causes individual fluid elements to migrate. Such a calculation has been carried out by Dore [5] in an extreme case of a stratified fluid, namely that of a two-layer system of finite depth. For maintained internal waves in a continuously-stratified fluid, we proceed as follows. Beyond the oscillatory boundary layers, adjacent to the free surface and horizontal bottom, Eq. (4.1) yields

$$
-\partial\left(\overline{\zeta_{1} \nabla^{2} \omega_{1}}\right) / \partial z=\partial^{2} \bar{\omega}_{2} / \partial z^{2}
$$




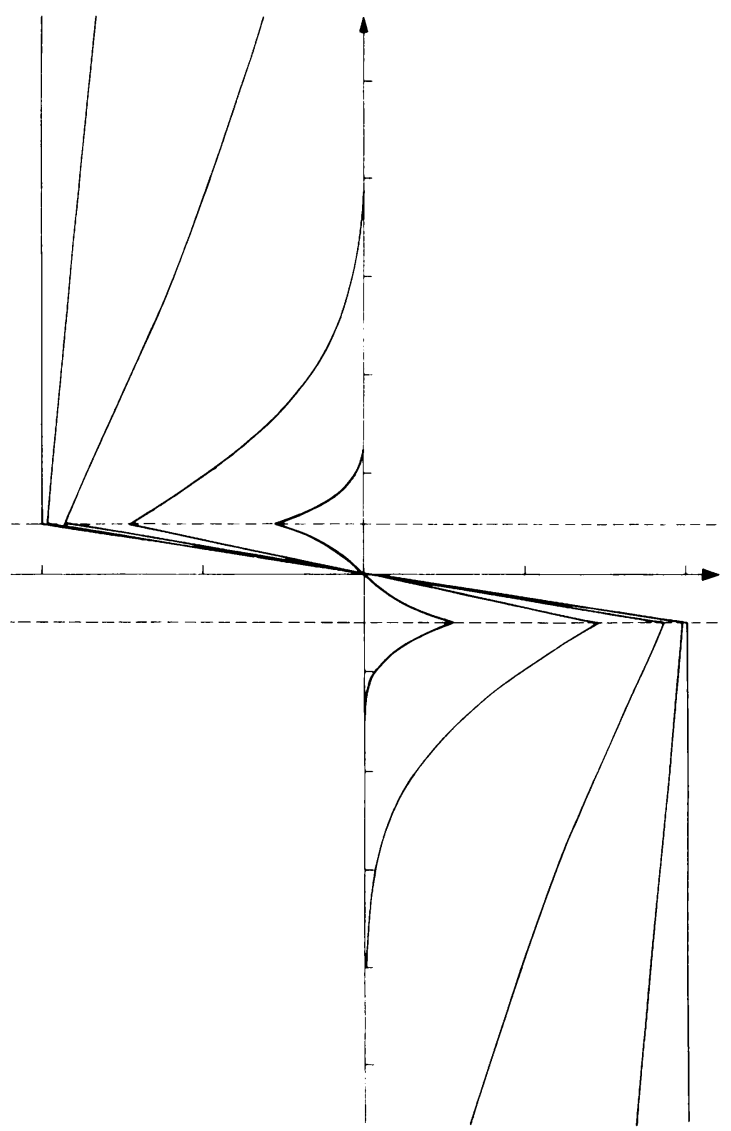

FIG. 2. Instantaneous distributions of secondary vorticity associated with the first internal mode on a thin, deep thermocline occupying the region $\frac{b}{2} b>z>-\frac{1}{2} b$.

in the steady state. Now the mean part of the buoyancy term $G \partial \bar{\rho}_{2} / \partial x$ has been assumed to be zero ${ }^{3}$ at $O\left(\alpha^{2} \epsilon^{2}\right)$, whence, by the $z$-component of the equation of motion and use of the time-averaged normal stress condition on the free surface, there can be no mean, horizontal pressure gradient $\partial \bar{p}_{2} / \partial x$ at $O\left(\alpha^{2} \epsilon^{2}\right)$. Thus, the $x$-component of the equation of motion yields

$$
\partial^{2} \bar{u}_{2} / \partial z^{2}=\frac{1}{2} \Psi\left[\left(d^{2} / d z^{2}\right)-1\right]\left(\left(N^{2} / \sigma^{2}\right) \Psi\right) .
$$

The integration of Eq. (5.2) is subject to boundary conditions at the top and bottom. Now, to the present order of accuracy, the top and bottom boundary layers may be regarded as locally homogeneous so that, referring to Eqs. (189), (227) of Longuet-Higgins [3], we deduce the following conditions on $\bar{u}_{2}$ :

$$
\begin{array}{ll}
\bar{u}_{2}=\frac{3}{4} \Psi^{\prime 2} & \text { on } z=-h, \\
\bar{\omega}_{2}=\partial \bar{u}_{2} / \partial z=0 & \text { on } z=0 .
\end{array}
$$

${ }^{3}$ For, otherwise, $\bar{\rho}_{2}$ contains a term which is linear in $x$. This would imply that the mean positions of surfaces of constant density are tilted at arbitrary angles $O\left(\alpha^{2} \epsilon^{2}\right)$ with the horizontal. 
We then obtain

$$
\bar{u}_{2}=\frac{3}{4}\left[\Psi^{\prime}(-h)\right]^{2}+\frac{1}{2} \frac{N^{2}}{\sigma^{2}} \Psi^{2}-\int_{-h}^{z} \frac{N^{2}}{\sigma^{2}} \Psi \Psi^{\prime} d Z+\frac{1}{2} \int_{-h}^{z} \int_{z^{\prime}}^{0} \frac{N^{4}}{\sigma^{4}} \Psi^{2} d z^{\prime} d Z,
$$

giving the second-order, Eulerian velocity at a point fixed in space. The horizontal mass transport velocity is given by the sum of this expression and that for the corresponding Stokes drift velocity, Eq. (2.7), so that

$$
\begin{aligned}
U_{l} & =\bar{u}_{2}+U_{s}=\bar{u}_{2}+\frac{1}{2}\left[\Psi^{\prime 2}-\left(N^{2} / \sigma^{2}-1\right) \Psi^{2}\right], \\
W_{l} & =\bar{w}_{2}+W_{s}=0 .
\end{aligned}
$$

The net mass flow across any section $x=$ constant is uniquely determined in this solution. This feature arises due to our insistence that the buoyancy term plays no influential part (see also Kelly [13, p. 143]), or, equivalently, that the mean position of isopycnal surfaces should be horizontal. The uniqueness property may be contrasted with the works on surface waves in homogeneous fluids by Longuet-Higgins [3], Ünlüata and Mei [14], and Mei, Liu and Carter [15], each of which allows a mean, horizontal pressure gradient $O\left(\alpha^{2} \epsilon^{2}\right)$, with a consequent tilt of the mean position of the free surface.

The results of Eqs. (5.5), (5.6) may be simply illustrated for a uniformly-stratified fluid, for which

$$
\Psi=A \sin \frac{n \pi(z+h)}{h} \quad(n=1,2, \cdots),
$$

and $N^{2} / \sigma^{2}=1+n^{2} \pi^{2} / h^{2}$. The net mass flow is found to be positive for all internal modes $n$ $=1,2, \cdots$. The profile of $U_{l}$ for the first internal mode $(n=1)$ is compared in Fig. 3 with its counterpart from inviscid theory, which comprises $U_{s}$ alone, is symmetrical about $z=$ $-\frac{1}{2} h$, and has zero net mass flow.

Appendix 1. We derive the partial differential equation governing the contribution

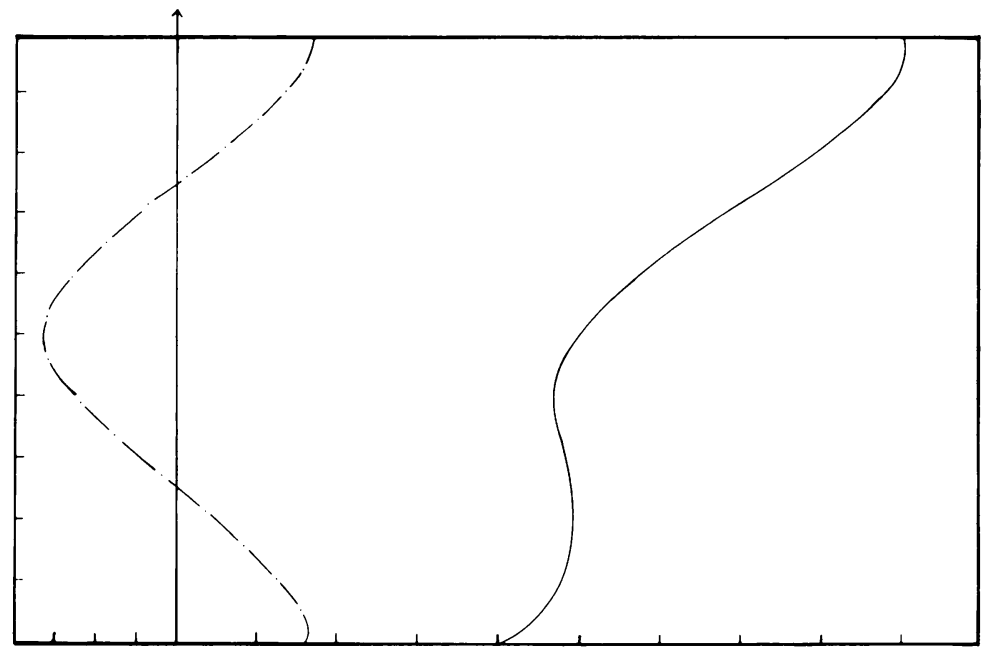

FIG. 3. Profiles of the mass transport velocity ( - ) and the Stokes drift velocity ( $--\longrightarrow$ ) for the first internal mode in a uniformly-stratified fluid. 
$\omega_{m}(z, t)$ of the secondary vorticity $\omega_{2}(x, z, t)$-see Eq. (4.6)-when the internal wave is maintained both spatially and temporally. By Eq. (4.1),

$$
\frac{\partial \omega_{2}}{\partial t}+\frac{\partial}{\partial x}\left(u_{1} \omega_{1}\right)+\frac{\partial}{\partial z}\left(w_{1} \omega_{1}\right)=\epsilon^{2} \nabla^{2} \omega_{2}+G \frac{\partial \rho_{2}}{\partial x}
$$

for all $t>0$ for sufficiently small wave amplitudes such that $\alpha<\epsilon$; for larger amplitudes, the terms here should still be the dominant terms of Eq. (4.1) for the initial growth of secondary vorticity $\omega_{m}$ in regions of stratification and in homogeneous fluid adjacent to such regions-see also Longuet-Higgins [1, p. 302]. Now, we have no vertical component of velocity associated with $\omega_{m}$, so that the buoyancy term in (A1.1) cannot be influential in determining $\omega_{m}$. Further, since $u_{1}, w_{1}, \omega_{1}$ have the form $U(z) \exp (i(k x-\sigma t))$, etc., we have

$$
\partial \omega_{m} / \partial t+\partial\left(\overline{w_{1} \omega_{1}}\right) / \partial z=\epsilon^{2} \partial^{2} \omega_{m} / \partial z^{2} .
$$

But, if $\zeta$ denotes the vertical displacement of a fluid element,

$$
\overline{w_{1} \omega_{1}}=\overline{\omega_{1} \partial \zeta_{1} / \partial t}=-\overline{\zeta_{1} \partial \omega_{1} / \partial t} .
$$

Now Eqs. (4.1), (4.2) give

$$
\partial \omega_{1} / \partial t=\epsilon^{2} \nabla^{2} \omega_{1}+G \partial \rho_{1} / \partial x, \quad \partial \rho_{1} / \partial t+\rho_{0}^{\prime} \partial \zeta_{1} / \partial t=0,
$$

respectively, and so, since $\partial \rho_{1} / \partial x=-\partial \rho_{1} / \partial t$,

$$
\overline{w_{1} \omega_{1}}=-\overline{\zeta_{1}\left[\epsilon^{2} \nabla^{2} \omega_{1}+G \rho_{0}^{\prime} \partial \zeta_{1} / \partial t\right]}=-\epsilon^{2} \overline{\zeta_{1} \nabla^{2} \omega_{1}} .
$$

Combining Eqs. (A1.2), (A 1.3) gives the following equation governing the development of $\omega_{m}$ :

$$
\partial \omega_{m} / \partial t-\epsilon^{2} \frac{\partial}{\partial z} \overline{\left(\zeta_{1} \nabla^{2} \omega_{1}\right)}=\epsilon^{2}\left(\partial^{2} \omega_{m} / \partial z^{2}\right)
$$

which is Eq. (4.7) of the text. The non-homogeneous term in this equation clearly arises from the presence of the Reynolds stress $-\overline{u_{1} w_{1}}$ in Eq. (A1.2).

Appendix 2. When the internal wave is assumed to decay with time, it is found that Eq. (A1.4) is significantly modified. The quantities $u_{1}, w_{1}, \omega_{1}$ now have the form $U(z)$ exp $\left(i\left(k x-\sigma_{R} t\right)\right) \exp (-t / \tau)$, etc., where $2 \pi / \sigma_{R}$ is the quasi-period and $\tau=O\left(\epsilon^{-2}\right)$ is the inverse logarithmic decrement of the decaying motion. Eq. (A1.2) still holds, but we now have

$$
\begin{aligned}
\overline{w_{1} \omega_{1}} & =-\overline{\zeta_{1} \partial \omega_{1} / \partial t}+\overline{\partial\left(\zeta_{1} \omega_{1}\right) / \partial t}=-\overline{\zeta_{1}\left[\epsilon^{2} \nabla^{2} \omega_{1}+G \rho_{0}^{\prime} \partial \zeta_{1} / \partial x\right]}+\overline{\partial\left(\zeta_{1} G \rho_{0}^{\prime} \zeta_{1}\right) \partial t}+O\left(\epsilon^{4}\right) \\
& \simeq-\epsilon^{2} \overline{\zeta_{1} \nabla^{2} \omega_{1}}-2 G \tau^{-1} \rho_{0}^{\prime} \Psi^{2} \exp (-2 t / \tau) .
\end{aligned}
$$

Thus, Eq. (A1.2) becomes

$$
\frac{\partial \omega_{m}}{\partial t}-\epsilon^{2} \frac{\partial}{\partial z}\left(\overline{\zeta_{1} \nabla^{2} \omega_{1}}\right)-2 G \tau^{-1}\left(\rho_{0}^{\prime} \Psi^{2}\right)^{\prime} \exp (-2 t / \tau)=\epsilon^{2} \frac{\partial^{2} \omega_{m}}{\partial z^{2}}
$$

for the decaying wave, as in Eq. (4.24) of the text.

Note added in proof. Recent work by Dore [16] for progressive surface waves shows that the effect of air drag is usually important, except for short waves. A similar effect may 
possibly be expected at the air-water interface for sufficiently long internal waves, as in Eq. (4.3) and Sec. 4(i). No modification arises, though, in Secs. 3 and 4(ii).

\section{REFERENCES}

[1] M. S. Longuet-Higgins, Mass transport in the boundary layer at a free oscillating surface, J. Fluid Mech. 1960

[2] O. M. Phillips, The dynamics of the upper ocean, Cambridge University Press, 1966

[3] M. S. Longuet-Higgins, Mass transport in water waves, Phil. Trans. Roy. Soc. Lond. 1953

[4] M. S. Longuet-Higgins, A nonlinear mechanism for the generation of sea waves, Proc. Roy. Soc. Lond. 1969

[5] B. D. Dore, Mass transport in layered fuid systems, J. Fluid Mech. 1970

[6] B. D. Dore, A contribution to the theory of viscous damping of stratified wave flows, Acta Mech. 1969

[7] S. A. Thorpe, On the shape of progressive internal waves, Phil. Trans. Roy. Soc. Lond., 1968

[8] H. S. Carslaw and J. C. Jaeger, Conduction of heat in solids, Oxford University Press (2nd ed.), 1959

[9] M. A. Al-Zanaidi and B. D. Dore, Some aspects of internal wave motions, Pure and Appl. Geophys. 1976

[10] M. A. Al-Zanaidi, Some aspects of internal wave motions, M. Phil. Dissertation, University of Reading, 1975

[11] B. D. Dore, Viscous damping of small amplitude waves in a non-homogeneous fluid of infinite depth. Deep-Sea Res. 1968

[12] M. Abramowitz and I. A. Stegun, Handbook of mathematical functions, Dover Publications, 1965

[13] R. E. Kelly, Wave-induced boundary layers in a stratified fluid, J. Fluid Mech. 1970

[14] U. Ünlüata and C. C. Mei, Mass transport in water waves, J. Geophys. Res. 1970

[15] C. C. Mei, P. L.-F. Liu and T. G. Carter, Mass transport in water waves, Ralph M. Parsons Laboratory for Water Resources and Hydrodynamics, Massachusetts Institute of Technology, Report 146, 1972

[16] B. O. Dore, Some effects of the air-water interface on gravity waves, Geophys. Astrophys. Fluid Dyn. 1978 